\title{
A COMPLETE CLASSIFICATION OF 3-DIMENSIONAL QUADRATIC AS-REGULAR ALGEBRAS OF TYPE EC
}

\author{
MASAKI MATSUNO
}

\begin{abstract}
Classification of AS-regular algebras is one of the main interests in noncommutative algebraic geometry. We say that a 3dimensional quadratic AS-regular algebra is of Type EC if its point scheme is an elliptic curve in $\mathbb{P}^{2}$. In this paper, we give a complete list of geometric pairs and a complete list of twisted superpotentials corresponding to such algebras. As an application, we show that there are only two exceptions up to isomorphism among all 3-dimensional quadratic AS-regular algebras which cannot be written as a twist of a Calabi-Yau AS-regular algebra by a graded algebra automorphism.
\end{abstract}

\section{INTRODUCTUION}

In noncommutative algebraic geometry, an Artin-Schelter regular (ASregular) algebra defined by Artin-Schelter [1] is one of the most important classes of algebras to study. Classification of AS-regular algebras is one of the important problems in this field. In fact, Artin-Tate-Van den Bergh [2] proved that there exists a one-to-one correspondence between 3-dimensional AS-regular algebras and regular geometric pairs by using algebraic geometry, which was a starting point of noncommutative algebraic geometry. In this paper, we say that a 3-dimensional quadratic AS-regular algebra is of Type EC (or simply a Type EC algebra) if its point scheme is an elliptic curve in $\mathbb{P}^{2}$. A typical example is a 3-dimensional Sklyanin algebra (Type A algebra), which had been intensively studied in many literature, but there are others (Type B, E, H algebras), which have been much less paid attention to.

A list of geometric pairs for "generic" 3-dimensional AS-regular algebras was given in $[2,4.13]$. It is known that there are many "non-generic" 3 dimensional AS-regular algebras (see, for example, [9, Theorem 3.1]). Restricting to Type EC algebras, we have the following questions:

(1) Is there a "non-generic" 3-dimensional AS-regular algebra of Type EC?

(2) Is every geometric pair given in the list of $[2,4.13]$ in fact regular?

(3) How many algebras in each type up to isomorphism?

To resolve these questions, we redefine Type B, E, $\mathrm{H}$ algebras in this paper and give a more complete list of geometric pairs for Type EC algebras. In

Date: December 17, 2019.

2010 Mathematics Subject Classification. 14A22, 14H52, 16W50, 16S37, 16E65.

Key words and phrases. AS-regular algebras, Geometric algebras, Calabi-Yau algebras, Superpotentials, Elliptic curves. 
particular, we show that there are only two Type E algebras and only two Type $\mathrm{H}$ algebras up to isomorphism.

Recently, Dubois-Violette [5] and Bocklandt-Schedler-Wemyss [4] showed that every 3-dimensional quadratic AS-regular algebra $A$ is isomorphic to a derivation-quotient algebra $\mathcal{D}(w)$ of a twisted superpotential $w$, and MoriSmith [14] classified 3-dimensional quadratic Calabi-Yau AS-regular algebras by using superpotentials. In this paper, we give a list of twisted superpotentials for Type EC algebras. Combining with the list of twisted superpotentials for non-Type EC algebras given in [10, Table 1], we finally complete the project started by [1], the classification of 3-dimensional AS-regular algebras up to isomorphism, in the quadratic case.

It was proved in [10, Corollary 3.7] that every non-Type EC algebra can be written as a twist of a Calabi-Yau AS-regular algebra by a graded algebra automorphism. As an application of our classification, we show that there are only two exceptions up to isomorphism among all 3-dimensional quadratic AS-regular algebras for this property to hold:

Theorem 1.1 (Theorem 4.8). A 3-dimensional quadratic AS-regular algebra is a twist of a Calabi-Yau AS-regular algebra by a graded algebra automorphism if and only if it is not of Type E.

This paper is organized as follows: In section 2, we recall the definitions of an AS-regular algebra from [1], a Calabi-Yau algebra from [7], a twisted superpotential and a twist of a superpotential in the sense of [13]. Also, we recall the definitions of a geometric algebra for quadratic algebras from [12]. We also recall some properties of elliptic curves in $\mathbb{P}^{2}$ and the results of the previous paper [9]. In section 3, we give a complete list of geometric pairs for Type EC algebras up to isomorphism (Theorem 3.13, Table 1). Finally, in section 4, we give a complete list of twisted superpotentials for Type EC algebras up to isomorphism (Theorem 4.2, Table 2) and we prove Theorem 1.1 .

\section{Preliminaries}

Throughout this paper, let $k$ be an algebraically closed field of characteristic 0 . A graded $k$-algebra means an $\mathbb{N}$-graded algebra $A=\bigoplus_{i \in \mathbb{N}} A_{i}$. A connected graded $k$-algebra $A$ is a graded $k$-algebra such that $A_{0}=k$.

2.1. AS-regular algebras and Calabi-Yau algebras. Let $A$ be a connected graded $k$-algebra. The Gelfand-Kirillov dimension of $A$ is defined by $\operatorname{GKdim} A:=\inf \left\{\alpha \in \mathbb{R} \mid \operatorname{dim}_{k}\left(\sum_{i=0}^{n} A_{i}\right) \leq n^{\alpha}\right.$ for all $\left.n \gg 0\right\}$.

Definition 2.1 ([1]). A connected graded algebra $A$ is called a $d$-dimensional Artin-Schelter regular ( $A S$-regular) algebra if $A$ satisfies the following conditions:

(i) $\operatorname{gldim} A=d<\infty$,

(ii) $\operatorname{GKdim} A<\infty$, 
(iii) (Gorenstein condition) $\operatorname{Ext}_{A}^{i}(k, A) \cong \begin{cases}k & (i=d), \\ 0 & (i \neq d) .\end{cases}$

Any 3-dimensional AS-regular algebra $A$ generated in degree 1 is either a quadratic or a cubic algebra ([1, Theorem 1.5 (i)]). In this paper, we focus on 3-dimensional quadratic AS-regular algebras.

Here, we recall the definition of a Calabi-Yau algebra introduced by [7].

Definition $2.2([7])$. A $k$-algebra $S$ is called $d$-dimensional Calabi-Yau if $S$ satisfies the following conditions:

(i) $\operatorname{pd}_{S^{\mathrm{e}}} S=d<\infty$,

(ii) $\operatorname{Ext}_{S^{\mathrm{e}}}^{i}\left(S, S^{\mathrm{e}}\right) \cong\left\{\begin{array}{ll}S & \text { if } i=d, \\ 0 & \text { if } i \neq d\end{array} \quad\right.$ (as $S^{\mathrm{e}}$-modules)

where $S^{\mathrm{e}}=S \otimes_{k} S^{\mathrm{op}}$ is the enveloping algebra of $S$.

Remark 2.3 ([16, Lemma 1.2]). Let $A$ be a connected graded $k$-algebra of finite GK-dimension. If $A$ is a $d$-dimensional Calabi-Yau algebra, then it is a $d$-dimensional AS-regular algebra.

2.2. Geometric algebras. Let $V$ be a finite dimensional $k$-vector space. For a quadratic algebra $A=T(V) /(R)$ where $R \subset V \otimes_{k} V$, we set

$$
\mathcal{V}(R):=\left\{(p, q) \in \mathbb{P}\left(V^{*}\right) \times \mathbb{P}\left(V^{*}\right) \mid f(p, q)=0 \text { for all } f \in R\right\} .
$$

In [12], Mori introduced a geometric algebra over $k$ as follows.

Definition $2.4([12])$. A geometric pair $(E, \sigma)$ consists of a projective variety $E \subset \mathbb{P}\left(V^{*}\right)$ and $\sigma \in$ Aut $_{k} E$. Let $A=T(V) /(R)$ be a quadratic algebra with $R \subset V \otimes_{k} V$.

(1) We say that $A$ satisfies (G1) if there exists a geometric pair $(E, \sigma)$ such that

$$
\mathcal{V}(R)=\left\{(p, \sigma(p)) \in \mathbb{P}\left(V^{*}\right) \times \mathbb{P}\left(V^{*}\right) \mid p \in E\right\} .
$$

In this case, we write $\mathcal{P}(A)=(E, \sigma)$.

(2) We say that $A$ satisfies (G2) if there exists a geometric pair $(E, \sigma)$ such that

$$
R=\left\{f \in V \otimes_{k} V \mid f(p, \sigma(p))=0 \text { for any } p \in E\right\} .
$$

In this case, we write $A=\mathcal{A}(E, \sigma)$.

(3) A quadratic algebra $A$ is called geometric if $A$ satisfies both (G1) and (G2) with $A=\mathcal{A}(\mathcal{P}(A))$.

When $A=\mathcal{A}(E, \sigma)$ is a geometric algebra, $E$ is called the point scheme of A.

Theorem 2.5 ([2]). Every 3-dimensional quadratic AS-regular algebra $A$ is geometric. Moreover, the point scheme $E$ of $A$ is either $\mathbb{P}^{2}$ or a cubic divisor in $\mathbb{P}^{2}$.

Remark 2.6. In the above theorem, $E \subset \mathbb{P}^{2}$ could be a non-reduced cubic divisor in $\mathbb{P}^{2}$. See [12, Definition 4.3$]$ for the definition of a geometric algebra in the case that $E$ is non-reduced. 
2.3. Derivation-quotient algebras. Now, we recall the definitions of a superpotential, a twisted superpotential and a derivation-quotient algebra from [4] and [13]. Also, we recall the definition of a twist of a superpotential due to [13].

Let $V$ be a 3 -dimensional $k$-vector space and we fix a basis $\left\{x_{1}, x_{2}, x_{3}\right\}$ for $V$. In this paper, we call an element $w \in V^{\otimes 3}$ a potential. For $w \in V^{\otimes 3}$, there exist unique $w_{i} \in V^{\otimes 2}$ such that $w=\sum_{i=1}^{3} x_{i} \otimes w_{i}$. Then the left partial derivative of $w$ with respect to $x_{i}(i=1,2,3)$ is

$$
\partial_{x_{i}}(w):=w_{i},
$$

and the derivation-quotient algebra of $w$ is

$$
\mathcal{D}(w):=T(V) /\left(\partial_{x_{1}}(w), \partial_{x_{2}}(w), \partial_{x_{3}}(w)\right) .
$$

Since, for $w \in V^{\otimes 3}$, there exist unique $w_{i}^{\prime} \in V^{\otimes 2}$ such that $w=\sum_{i=1}^{3} w_{i}^{\prime} \otimes x_{i}$, we can also define the right partial derivative of $w$ with respect to $x_{i}(i=$ $1,2,3)$ by

$$
(w) \partial_{x_{i}}:=w_{i}^{\prime}
$$

We define the $k$-linear map $\varphi: V^{\otimes 3} \longrightarrow V^{\otimes 3}$ by

$$
\varphi\left(v_{1} \otimes v_{2} \otimes v_{3}\right):=v_{3} \otimes v_{1} \otimes v_{2} .
$$

We write $\mathrm{GL}(V)$ for the general linear group of $V$.

Definition 2.7 ([4], [13]). Let $w \in V^{\otimes 3}$ be a potential.

(1) If $\varphi(w)=w$ for $w \in V^{\otimes 3}$, then $w$ is called a superpotential.

(2) If there exists $\theta \in \mathrm{GL}(V)$ such that

$$
\left(\theta \otimes \operatorname{id}_{V} \otimes \operatorname{id}_{V}\right) \varphi(w)=w,
$$

then $w$ is called a twisted superpotential.

Lemma 2.8 ([13, Theorem 4.13]). Let $w \in V^{\otimes 3}$ be a potential. Assume that the left derivatives $\partial_{x_{1}}(w), \partial_{x_{2}}(w), \partial_{x_{3}}(w)$ are linearly independent. Then $w$ is a twisted superpotential if and only if there is $Q \in \mathrm{GL}_{3}(k)$ such that

$$
\left((w) \partial_{x_{1}},(w) \partial_{x_{2}},(w) \partial_{x_{3}}\right)=Q\left(\partial_{x_{1}}(w), \partial_{x_{2}}(w), \partial_{x_{3}}(w)\right) .
$$

We recall the notion of twisted algebra by a graded algebra automorphism. We denote by $\mathrm{GrAut}_{k} A$ the group of graded algebra automorphisms of $A$.

Let $\phi \in \operatorname{GrAut}_{k} A$. A new graded and associative multiplication $*$ on the underlying graded $k$-vector space $\bigoplus_{i \in \mathbb{N}} A_{i}$ is defined by

$$
a * b:=a \phi^{l}(b) \text { for all } a \in A_{l}, b \in A_{m} .
$$

The graded $k$-algebra $\left(\bigoplus_{i \in \mathbb{N}} A_{i}, *\right)$ is called the twisted algebra of $A$ by $\phi$ and is denoted by $A^{\phi}$.

Definition 2.9 ([13]). For a superpotential $w \in V^{\otimes 3}$ and $\theta \in \operatorname{GL}(V)$,

$$
w^{\theta}:=\left(\theta^{2} \otimes \theta \otimes \operatorname{id}_{V}\right)(w)
$$

is called a Mori-Smith twist (MS twist) of $w$ by $\theta$. 
For a potential $w \in V^{\otimes 3}$, we define

$$
\operatorname{Aut}(w):=\left\{\theta \in \operatorname{GL}(V) \mid\left(\theta^{\otimes 3}\right)(w)=\lambda w, \exists \lambda \in k^{\times}\right\} .
$$

Lemma 2.10 ([13, Proposition 5.2]). For a superpotential $w \in V^{\otimes 3}$ and $\phi \in \operatorname{GrAut}_{k} \mathcal{D}(w)$, we have that $\left.\phi\right|_{V} \in \operatorname{Aut}(w)$ and $\mathcal{D}\left(w^{\left.\phi\right|_{V}}\right) \cong \mathcal{D}(w)^{\phi}$.

We say that a potential $w \in V^{\otimes 3}$ is regular (resp. Calabi-Yau) if the derivation-quotient algebra $\mathcal{D}(w)$ is a 3-dimensional quadratic AS-regular (resp. Calabi-Yau AS-regular) algebra.

Lemma 2.11 ([13, Corollary 4.5]). Let $w \in V^{\otimes 3}$ be a regular potential. Then $w$ is Calabi-Yau if and only if it is a superpotential.

Lemma 2.12 ([10, Lemma 2.16]). If $w \in V^{\otimes 3}$ is a regular superpotential and $\theta \in \operatorname{Aut}(w)$, then the $M S$ twist $w^{\theta}$ is a regular twisted superpotential.

Remark 2.13. If $\theta \in \mathrm{GL}(V) \backslash \operatorname{Aut}(w)$, then the MS twist $w^{\theta}$ of a regular superpotential $w \in V^{\otimes 3}$ by $\theta$ may not be regular nor a twisted superpotential (see [10, Remark 2.12]).

2.4. Elliptic curves in $\mathbb{P}^{2}$. For the rest of this paper, let $E$ be an elliptic curve in $\mathbb{P}^{2}$ and we use a Hesse form $E=\mathcal{V}\left(x^{3}+y^{3}+z^{3}-3 \lambda x y z\right)$ where $\lambda \in k$ with $\lambda^{3} \neq 1$. Note that every elliptic curve in $\mathbb{P}^{2}$ can be written in this form ([6, Corollary 2.18]). Our aim in this section is to recall some properties of elliptic curves in $\mathbb{P}^{2}$.

The $j$-invariant of a Hesse form is given by the following formula (see [6, Proposition 2.16]):

$$
j(E)=\frac{27 \lambda^{3}\left(\lambda^{3}+8\right)^{3}}{\left(\lambda^{3}-1\right)^{3}} .
$$

It is well-known that the $j$-invariant $j(E)$ classifies elliptic curves in $\mathbb{P}^{2}$ up to projective equivalence ([8, Theorem IV $4.1(\mathrm{~b})])$. For $o_{E}:=(1:-1: 0) \in E$, the group structure on $\left(E, o_{E}\right)$ is given as follows ([6, Theorem 2.11]): for $p=(a: b: c), q=(\alpha: \beta: \gamma) \in E$,

$$
p+q=\left\{\begin{array}{l}
\left(a c \beta^{2}-b^{2} \alpha \gamma: b c \alpha^{2}-a^{2} \beta \gamma: a b \gamma^{2}-c^{2} \alpha \beta\right) \text { or, } \\
\left(a b \alpha^{2}-c^{2} \beta \gamma: a c \gamma^{2}-b^{2} \alpha \beta: b c \beta^{2}-a^{2} \alpha \gamma\right) .
\end{array}\right.
$$

Throughout this paper, we fix the group structure on $E$ with the zero element $o_{E}:=(1:-1: 0) \in E$.

We define

$$
\begin{aligned}
& \operatorname{Aut}_{k}\left(\mathbb{P}^{2}, E\right):=\left\{\sigma \in \operatorname{Aut}_{k} \mathbb{P}^{2}|\sigma|_{E} \in \operatorname{Aut}_{k} E\right\}, \\
& \operatorname{Aut}_{k}\left(E, o_{E}\right):=\left\{\sigma \in \operatorname{Aut}_{k} E \mid \sigma\left(o_{E}\right)=o_{E}\right\} .
\end{aligned}
$$

It is well-known that $\operatorname{Aut}_{k}\left(E, o_{E}\right)$ is a finite cyclic subgroup of $\operatorname{Aut}_{k} E$ ([8, Corollary IV 4.7]). A generator of $\operatorname{Aut}_{k}\left(E, o_{E}\right)$ is given in the next lemma.

Lemma 2.14 ([9, Theorem 4.6]). A generator $\tau$ of $\operatorname{Aut}_{k}\left(E, o_{E}\right)$ is given by (i) $\tau_{1}(a: b: c):=(b: a: c)$ if $j(E) \neq 0,12^{3}$, 
(ii) $\tau_{2}(a: b: c):=(b: a: c \varepsilon)$ if $\lambda=0$ (so that $\left.j(E)=0\right)$,

(iii) $\tau_{3}(a: b: c):=\left(a \varepsilon^{2}+b \varepsilon+c: a \varepsilon+b \varepsilon^{2}+c: a+b+c\right)$ if $\lambda=1+\sqrt{3}$ (so that $\left.j(E)=12^{3}\right)$

where $\varepsilon$ is a primitive 3 rd root of unity. In particular, $\operatorname{Aut}_{k}\left(E, o_{E}\right)$ is a subgroup of $\operatorname{Aut}_{k}\left(\mathbb{P}^{2}, E\right)$.

Note that $\left|\tau_{1}\right|=2,\left|\tau_{2}\right|=6$ and $\left|\tau_{3}\right|=4$. Since $\tau_{2}^{3}=\tau_{3}^{2}=\tau_{1}$, we use the notation $\tau_{1} \in \operatorname{Aut}_{k}\left(\mathbb{P}^{2}, E\right)$ even when $j(E)=0,12^{3}$.

Remark 2.15. When $j(E)=0,12^{3}$, we fix $\lambda=0,1+\sqrt{3}$ respectively as in Lemma 2.14 to classify $\mathcal{A}(E, \sigma)$ up to graded algebra isomorphism, because if $E$ and $E^{\prime}$ are projectively equivalent, then, for any $\mathcal{A}(E, \sigma)$, there is an automorphism $\sigma^{\prime} \in \mathrm{Aut}_{k} E^{\prime}$ such that $\mathcal{A}(E, \sigma) \cong \mathcal{A}\left(E^{\prime}, \sigma^{\prime}\right)$ (see [15, Lemma $2.6])$.

Every automorphism $\sigma \in \operatorname{Aut}_{k} E$ can be written as $\sigma=\sigma_{p} \tau^{i}$ where $\sigma_{p}$ is a translation by a point $p \in E, \tau$ is a generator of $\operatorname{Aut}_{k}\left(E, o_{E}\right)$ and $i \in \mathbb{Z}_{|\tau|}$ ([9, Proposition 4.5]).

For any $n \geq 1$, a point $p \in E$ is called $n$-torsion if

$$
n p:=\underbrace{p+\cdots+p}_{n}=o_{E} .
$$

We set $E[n]:=\left\{p \in E \mid n p=o_{E}\right\}$. Note that $E[n]$ is a subgroup of $E$ and $|E[n]|=n^{2}([8$, Example 4.8.1]).

We use the following easy lemma several times.

Lemma 2.16. Let $E=\mathcal{V}\left(x^{3}+y^{3}+z^{3}-3 \lambda x y z\right)$ be an elliptic curve in $\mathbb{P}^{2}$ where $\lambda \in k$ with $\lambda^{3} \neq 1$.

(1) $E[6]=E[2] \oplus E[3]$.

(2) For $p=(a: b: c) \in E$, the inverse element of $p$ is $\tau_{1}(p)=(b: a: c)$.

(3) For $p=(a: b: c) \in E, p \in E[3]$ if and only if $a b c=0$.

(4) For $p=(a: b: c) \in E \backslash E[3], p \in E[2]$ if and only if $a=b$.

\section{Geometric pairs for Type EC algebras}

We say that a geometric algebra $\mathcal{A}(E, \sigma)$ is of Type $E C$ if $E$ is an elliptic curve in $\mathbb{P}^{2}$. For a quadratic algebra $\mathcal{A}\left(E, \sigma_{p} \tau^{i}\right)$ where $p \in E,\langle\tau\rangle=$ $\operatorname{Aut}_{k}\left(\mathbb{P}^{2}, E\right)$ and $i \in \mathbb{Z}_{|\tau|}, \mathcal{A}\left(E, \sigma_{p} \tau^{i}\right)$ is a geometric algebra of Type EC if and only if $p \in E \backslash E[3]([9$, Lemma 4.14]). In the previous paper [9], we gave a simple condition when two geometric algebras of Type EC are isomorphic. For each $i \in \mathbb{Z}_{|\tau|}$, we define

$$
U^{\tau^{i}}:=\left\{r-\tau^{i}(r) \mid r \in E[3]\right\} .
$$

Theorem 3.1 ([9, Theorem 4.16]). Let $E$ be an elliptic curve in $\mathbb{P}^{2}, p, q \in$ $E \backslash E[3]$ and $i, j \in \mathbb{Z}_{|\tau|}$. Then $\mathcal{A}\left(E, \sigma_{p} \tau^{i}\right) \cong \mathcal{A}\left(E, \sigma_{q} \tau^{j}\right)$ if and only if $i=j$ and $q=\tau^{l}(p)+r$ where $r \in U^{\tau^{i}}$ and $l \in \mathbb{Z}_{|\tau|}$. 
Lemma $3.2([9$, Lemma 4.17]).

$$
U^{\tau^{i}}= \begin{cases}\left\{o_{E}\right\} & \text { if } i=0, \\ \langle(1:-\varepsilon: 0)\rangle & \text { if } \lambda=0 \text { and } i=2,4, \\ E[3] & \text { otherwise }\end{cases}
$$

where $\varepsilon$ is a primitive 3 rd root of unity.

In the previous paper [10], we gave a simple condition to determine whether a geometric algebra of Type EC is AS-regular. For each $i \in \mathbb{Z}_{|\tau|}$, we define

$$
U_{\tau^{i}}:=\left\{p \in E \mid p-\tau^{i}(p) \in E[3]\right\} .
$$

Theorem 3.3 ([10, Theorem 4.3]). For $p \in E$ and $i \in \mathbb{Z}_{|\tau|}, \mathcal{A}\left(E, \sigma_{p} \tau^{i}\right)$ is a 3-dimensional quadratic AS-regular algebra if and only if $p \in U_{\tau^{i}} \backslash E[3]$.

Since $U_{\tau^{0}}=E, \mathcal{A}\left(E, \sigma_{p}\right)$ is AS-regular if and only if $p \in E \backslash E[3]$. A 3dimensional quadratic AS-regular algebra $\mathcal{A}\left(E, \sigma_{p}\right)$ is called a 3-dimensional Sklyanin algebra. Since $\tau$ is a group homomorphism of $E, E[3] \subset U_{\tau^{i}}$ for any $i \in \mathbb{Z}_{|\tau|}$.

For each $i \in \mathbb{Z}_{|\tau|}$, we define

$$
E_{\tau^{i}}:=\left\{p \in E \mid \tau^{i}(p)=p\right\} .
$$

It is easy to check the following lemma.

Lemma 3.4. (1) $E_{\tau^{0}}=E$.

(2) $E_{\tau_{1}}=E[2]$.

(3) For any $i \in \mathbb{Z}_{|\tau|}, E_{\tau^{i}}=E_{\tau|\tau|-i}$.

The next lemma is important to determine $U_{\tau^{i}}$.

Lemma 3.5. For any $i \in \mathbb{Z}_{|\tau|}$,

$$
U_{\tau^{i}}=\left\{p \in E \mid 3 p \in E_{\tau^{i}}\right\} .
$$

Proof. For any $i \in \mathbb{Z}_{|\tau|}, p \in U_{\tau^{i}}$ if and only if $p-\tau^{i}(p) \in E[3]$. Since $\tau$ is a group homomorphism, $p-\tau^{i}(p) \in E[3]$ if and only if $\tau^{i}(3 p)=3 p$. Hence $p \in U_{\tau^{i}}$ if and only if $3 p \in E_{\tau^{i}}$.

Proposition 3.6. (1) If $j(E) \neq 0,12^{3}$, then

$$
E_{\tau_{1}^{i}}= \begin{cases}E & \text { if } i=0, \\ E[2] & \text { if } i=1 .\end{cases}
$$

(2) If $\lambda=0$, then

$$
E_{\tau_{2}^{i}}= \begin{cases}E & \text { if } i=0, \\ \left\{o_{E}\right\} & \text { if } i=1,5, \\ \langle(1:-\varepsilon: 0)\rangle & \text { if } i=2,4, \\ E[2] & \text { if } i=3 .\end{cases}
$$

where $\varepsilon$ is a primitive 3 rd root of unity. 
(3) If $\lambda=1+\sqrt{3}$, then

$$
E_{\tau_{3}^{i}}= \begin{cases}E & \text { if } i=0, \\ \langle(1: 1: \lambda)\rangle & \text { if } i=1,3, \\ E[2] & \text { if } i=2 .\end{cases}
$$

Proof. (1) By Lemma 3.4, the formulas hold.

(2) Assume that $\lambda=0$. By Lemma 3.4, it is sufficient to prove the cases $i=1,2$.

(i) If $i=1$, then $p=(a: b: c) \in E_{\tau_{2}}$ if and only if $(b: a: c \varepsilon)=$ $(a: b: c)$. If $c=0$, then there is $\alpha \in k^{\times}$such that $a=\alpha b$ and $b=\alpha a$, so we have that $p=(1: 1: 0)$ or $p=(1:-1: 0)=o_{E}$. Since $(1: 1: 0) \notin E$, it follows that $p=o_{E}$. If $c \neq 0$, then $a=\varepsilon b$ and $b=\varepsilon a$. Since $\varepsilon$ is a primitive 3 rd root of unity, we have that $a=b=0$ but $(0: 0: 1) \notin E$. Hence $E_{\tau_{2}}=\left\{o_{E}\right\}$.

(ii) If $i=2$, then $p=(a: b: c) \in E_{\tau_{2}^{2}}$ if and only if $\left(a: b: c \varepsilon^{2}\right)=$ $(a: b: c)$. If $c \neq 0$, then since $a=\varepsilon^{2} a$ and $b=\varepsilon^{2} b$, it follows that $a=b=0$ but $(0: 0: 1) \notin E$. If $c=0$, then $a^{3}+b^{3}=0$, so $p=o_{E},(1:-\varepsilon: 0),\left(1:-\varepsilon^{2}: 0\right)$. Hence $E_{\tau_{2}^{2}}=\langle(1:-\varepsilon: 0)\rangle$.

(3) Assume that $\lambda=1+\sqrt{3}$. By Lemma 3.4, it is sufficient to prove the case $i=1$. If $p=(a: b: c) \in E_{\tau_{3}}$, then since $\tau_{3}^{2}(p)=\tau_{3}(p)=p$, it follows that $p \in E_{\tau_{3}^{2}}=E[2]$ by Lemma 3.4. Let $p \in E_{\tau_{3}} \backslash\left\{o_{E}\right\}$. By Lemma 3.4, $a=b$, so we assume that $p=(1: 1: c)$. Since $\tau_{3}(p)=$ $\left(\varepsilon^{2}+\varepsilon+c: \varepsilon+\varepsilon^{2}+c: 1+1+c\right)=(1: 1: c)$, there is $\alpha \in k^{\times}$such that $c-1=\alpha$ and $c+2=\alpha c$. Then we have that

$$
c^{2}-2 c-2=0 .
$$

By solving this equation, we have that $c=1 \pm \sqrt{3}$. Since $(1: 1: 1+\sqrt{3}) \in$ $E$ but $(1: 1: 1-\sqrt{3}) \notin E, E_{\tau_{3}} \leq\langle(1: 1: 1+\sqrt{3})\rangle$. Conversely,

$$
\begin{aligned}
& \tau_{3}(1: 1: 1+\sqrt{3})=(\sqrt{3}: \sqrt{3}: 3+\sqrt{3})=(1: 1: 1+\sqrt{3}), \\
& \text { so }\langle(1: 1: 1+\sqrt{3})\rangle \leq E_{\tau_{3}} \text {. Hence } E_{\tau_{3}}=\langle(1: 1: 1+\sqrt{3})\rangle .
\end{aligned}
$$

Lemma 3.7. If $E_{\tau^{i}} \leq E[2]$, then $U_{\tau^{i}}=E_{\tau^{i}} \oplus E[3]$.

Proof. Assume that $E_{\tau^{i}} \leq E[2]$. Since $E[2] \cap E[3]=\left\{o_{E}\right\}, E_{\tau^{i}} \cap E[3]=\left\{o_{E}\right\}$. If $p \in U_{\tau^{i}}$, then $3 p \in E_{\tau^{i}} \leq E[2]$ by Lemma 3.5, so $U_{\tau^{i}} \leq E[6]=E[2] \oplus E[3]$. For any $p \in U_{\tau^{i}}$, there are $q \in E[2]$ and $r \in E[3]$ such that $p=q+r$. Since $3 p \in E_{\tau^{i}}$,

$$
q=q+o_{E}=3 q+3 r=3 p \in E_{\tau^{i}},
$$

so we have that $U_{\tau^{i}} \leq E_{\tau^{i}} \oplus E[3]$. By definition of $E_{\tau^{i}}$, we have that $E_{\tau^{i}} \leq U_{\tau^{i}}$, so $E_{\tau^{i}} \oplus E[3] \leq U_{\tau^{i}}$. Hence $U_{\tau^{i}}=E_{\tau^{i}} \oplus E[3]$. 
Theorem 3.8. (1) If $j(E) \neq 0,12^{3}$, then

$$
U_{\tau_{1}^{i}}= \begin{cases}E & \text { if } i=0, \\ E[6] & \text { if } i=1 .\end{cases}
$$

(2) If $\lambda=0$, then

$$
U_{\tau_{2}^{i}}= \begin{cases}E & \text { if } i=0, \\ E[3] & \text { if } i=1,5, \\ E[3] \sqcup\left\{\tau_{2}^{l}(q)+r \mid l \in \mathbb{Z}_{6}, r \in E_{\tau_{2}^{2}}\right\} & \text { if } i=2,4, \\ E[6] & \text { if } i=3,\end{cases}
$$

where $q=\left(\eta^{8}: \eta^{4}: 1\right)$ and $\eta$ is a primitive 9 th root of unity.

(3) If $\lambda=1+\sqrt{3}$, then

$$
U_{\tau_{3}^{i}}= \begin{cases}E & \text { if } i=0, \\ \langle(1: 1: \lambda)\rangle \oplus E[3] & \text { if } i=1,3, \\ E[6] & \text { if } i=2 .\end{cases}
$$

Proof. For each case, if $i=0$, then $U_{\tau^{0}}=E$.

(1) If $i=1$, then $U_{\tau_{1}}=E[2] \oplus E[3]=E[6]$ by Proposition 3.6 (1) and Lemma 3.7.

(2) Assume that $\lambda=0$. Since $\tau_{2}^{3}=\tau_{1}$, it follows that $U_{\tau_{2}^{3}}=E[6]$. Since $U_{\tau_{2}^{i}}=U_{\tau_{2}^{6-i}}$, it is sufficient to prove the cases $i=1,2$.

If $i=1$, then $U_{\tau_{2}}=E[3]$ by Proposition 3.6 (2) and Lemma 3.7.

Assume that $i=2$. Let $p=(a: b: c) \in U_{\tau_{2}^{2}} \backslash E[3]$. Since $a b c \neq 0$, we may assume that $c=1$. Note that $p=(a: b: 1)$ satisfies $a^{3}+b^{3}+1=0$ and $a b \neq 0$. Since $3 p \in E_{\tau^{2}}$, the third component of $3 p$ is equal to 0 , that is,

$$
a b\left(b^{3}-a^{3}\right)^{2}-a b\left(a^{3}-1\right)\left(1-b^{3}\right)=0 .
$$

Since $b^{3}=-\left(a^{3}+1\right)$ and $a b \neq 0$, we have that $a^{6}+a^{3}+1=0$. Since $k$ is an algebraically closed field, the set of solutions is $\left\{\eta, \eta^{2}, \eta^{4}, \eta^{5}, \eta^{7}, \eta^{8}\right\}$ where $\eta$ is a primitive 9th root of unity. Then there are two cases (1) $a^{3}=\eta^{3}$ and (2) $a^{3}=\eta^{6}$. If $a^{3}=\eta^{3}$, then $b^{3}=\eta^{6}$, so $b=\eta^{2}, \eta^{5}, \eta^{8}$ and if $a^{3}=\eta^{6}$, then $b^{3}=\eta^{3}$, so $b=\eta, \eta^{4}, \eta^{7}$. Consequently, we have that

$U_{\tau_{2}^{2}} \subset E[3] \sqcup\left\{(a: b: 1) \mid\left(a^{3}, b^{3}\right)=\left(\eta^{3}, \eta^{6}\right)\right\} \sqcup\left\{(a: b: 1) \mid\left(a^{3}, b^{3}\right)=\left(\eta^{6}, \eta^{3}\right)\right\}$.

Conversely, if $p \in\left\{(a: b: 1) \mid\left(a^{3}, b^{3}\right)=\left(\eta^{3}, \eta^{6}\right)\right\}$, then

$$
2 p=\left(b: a \eta^{6}: \eta^{3}\right) \text { and } 3 p=\left(1:-\eta^{6}: 0\right) \in E_{\tau_{2}^{2}}
$$

by Proposition 3.6, so $p \in U_{\tau_{2}^{2}}$ by Lemma 3.5. Similarly, if

$$
p \in\left\{(a: b: 1) \mid\left(a^{3}, b^{3}\right)=\left(\eta^{6}, \eta^{3}\right)\right\},
$$

then

$$
2 p=\left(b \eta^{6}: a: \eta^{3}\right) \text { and } 3 p=\left(1:-\eta^{3}: 0\right) \in E_{\tau_{2}^{2}}
$$


so $p \in U_{\tau_{2}^{2}}$. Thus

$$
U_{2}=E[3] \sqcup\left\{(a: b: 1) \mid\left(a^{3}, b^{3}\right)=\left(\eta^{3}, \eta^{6}\right)\right\} \sqcup\left\{(a: b: 1) \mid\left(a^{3}, b^{3}\right)=\left(\eta^{6}, \eta^{3}\right)\right\} \text {. }
$$

Since

$$
\begin{aligned}
& \left\{\tau_{2}^{l}(q)+r \mid l \in \mathbb{Z}_{6}, r \in E_{\tau_{2}^{2}}\right\} \\
= & \left\{\left(\eta^{8}: \eta^{4}: 1\right),\left(\eta^{2}: \eta: 1\right),\left(\eta^{5}: \eta^{7}: 1\right),\left(\eta^{5}: \eta: 1\right),\left(\eta^{2}: \eta^{4}: 1\right),\left(\eta^{8}: \eta^{7}: 1\right),\right.
\end{aligned}
$$$$
\left(\eta^{5}: \eta^{4}: 1\right),\left(\eta^{8}: \eta: 1\right),\left(\eta^{2}: \eta^{7}: 1\right),\left(\eta: \eta^{5}: 1\right),\left(\eta^{4}: \eta^{2}: 1\right),\left(\eta^{7}: \eta^{8}: 1\right),
$$$$
\left.\left(\eta^{4}: \eta^{8}: 1\right),\left(\eta: \eta^{2}: 1\right),\left(\eta^{7}: \eta^{5}: 1\right),\left(\eta^{4}: \eta^{5}: 1\right),\left(\eta^{7}: \eta^{2}: 1\right),\left(\eta: \eta^{8}: 1\right)\right\} \text {, }
$$

we have that $U_{\tau_{2}^{2}}=E[3] \sqcup\left\{\tau_{2}^{l}(q)+r \mid l \in \mathbb{Z}_{6}, r \in E_{\tau_{2}^{2}}\right\}$.

(3) Assume that $\lambda=1+\sqrt{3}$. Since $\tau_{3}^{2}=\tau_{1}$, it follows that $U_{\tau_{3}^{2}}=E[6]$. Since $U_{\tau_{3}^{i}}=U_{\tau_{3}^{4-i}}$, it is sufficient to prove the case $i=1$.

If $i=1$, then $U_{\tau_{3}}=\langle(1: 1: \lambda)\rangle \oplus E[3]$ by Proposition $3.6(3)$ and Lemma 3.7.

Remark 3.9. By Theorem 3.8, if $\lambda=0$ and $i=1,5$, then a geometric algebra $\mathcal{A}\left(E, \sigma_{p} \tau_{2}^{i}\right)$ of Type EC is never AS-regular.

We define the four types of 3-dimensional quadratic AS-regular algebras $\mathcal{A}(E, \sigma)$ of Type EC mimicking [2, 4.13]:

(1) Type A: $\sigma=\sigma_{p}$.

(2) Type B: $\sigma=\sigma_{p} \tau_{1}$.

(3) Type E: $j(E)=0$ and $\sigma=\sigma_{p} \tau_{2}^{2}, \sigma_{p} \tau_{2}^{4}$.

(4) Type H: $j(E)=12^{3}$ and $\sigma=\sigma_{p} \tau_{3}, \sigma_{p} \tau_{3}^{3}$.

By Theorem 3.1, if $X \neq Y$, then Type $\mathrm{X}$ algebra is not isomorphic to any Type Y algebra.

Proposition 3.10. Fix an elliptic curve E.

(1) If $j(E) \neq 0,12^{3}$, then there are three Type $B$ algebras up to isomorphism.

(2) If $j(E)=0$, then there is one Type $B$ algebra up to isomorphism.

(3) If $j(E)=12^{3}$, then there are two Type $B$ algebras up to isomorphism.

Proof. Let $\mathcal{A}\left(E, \sigma_{p} \tau_{1}\right)$ be a Type B algebra where $p \in U_{\tau_{1}} \backslash E[3]$. By Theorem 3.8 , there are $q \in E[2]$ and $r \in E[3]$ such that $p=q+r$. Since $U^{\tau_{1}}=E[3]$ by Lemma 3.2, it follows from Theorem 3.1 that $\mathcal{A}\left(E, \sigma_{p} \tau_{1}\right) \cong \mathcal{A}\left(E, \sigma_{q} \tau_{1}\right)$, so we may assume that $p \in E[2] \backslash\left\{o_{E}\right\}$.

(1) Assume that $j(E) \neq 0,12^{3}$. Let $\mathcal{A}\left(E, \sigma_{p} \tau_{1}\right)$ and $\mathcal{A}\left(E, \sigma_{q} \tau_{1}\right)$ be Type $\mathrm{B}$ algebras where $p, q \in E[2] \backslash\left\{o_{E}\right\}$. By Theorem 3.1, $\mathcal{A}\left(E, \sigma_{p} \tau_{1}\right) \cong$ $\mathcal{A}\left(E, \sigma_{q} \tau_{1}\right)$ if and only if there exist $r \in U^{\tau_{1}}$ and $l \in \mathbb{Z}_{2}$ such that $q=\tau_{1}^{l}(p)+r$. Since $U^{\tau_{1}}=E[3]$ by Lemma 3.2 and $\tau_{1}(p)=p$, we have that $\mathcal{A}\left(E, \sigma_{p} \tau_{1}\right) \cong \mathcal{A}\left(E, \sigma_{p} \tau_{1}\right)$ if and only if $q-p \in E[3]$. Since $E[2] \cap E[3]=\left\{o_{E}\right\}, \mathcal{A}\left(E, \sigma_{p} \tau_{1}\right) \cong \mathcal{A}\left(E, \sigma_{q} \tau_{1}\right)$ if and only if $p=q$. It follows from $|E[2]|=2^{2}=4$ that there are three Type B algebras up to isomorphism. 
(2) Assume that $\lambda=0$. Let $p=(1: 1: c) \in E[2] \backslash\left\{o_{E}\right\}$. Since $E=$ $\mathcal{V}\left(x^{3}+y^{3}+z^{3}\right)$, we have that $c^{3}+2=0$, so

$$
c=-\sqrt[3]{2},-\sqrt[3]{2} \varepsilon,-\sqrt[3]{2} \varepsilon^{2}
$$

where $\varepsilon$ is a primitive 3 rd root of unity. We set $p_{1}=(1: 1:-\sqrt[3]{2}), p_{2}=$ $(1: 1:-\sqrt[3]{2} \varepsilon), p_{3}=\left(1: 1:-\sqrt[3]{2} \varepsilon^{2}\right)$. Since $p_{2}=\tau_{2}^{4}\left(p_{1}\right)$ and $p_{3}=\tau_{2}^{2}\left(p_{1}\right)$, we have that $\mathcal{A}\left(E, \sigma_{p_{1}} \tau_{2}^{3}\right) \cong \mathcal{A}\left(E, \sigma_{p_{2}} \tau_{2}^{3}\right) \cong \mathcal{A}\left(E, \sigma_{p_{3}} \tau_{2}^{3}\right)$ by Theorem 3.1. Thus there is one Type $\mathrm{B}$ algebra up to isomorphism.

(3) Assume that $\lambda=1+\sqrt{3}$. Let $p_{1}=(1: 1: \lambda)$. Since $E_{\tau_{3}}=\left\langle p_{1}\right\rangle$ by Proposition 3.6 and $U^{\tau_{3}^{2}}=E[3]$ by Lemma 3.2 ,

$$
\left\{\tau_{3}^{l}\left(p_{1}\right)+r \mid l \in \mathbb{Z}_{4}, r \in U^{\tau_{3}^{2}}\right\}=\left\{p_{1}+r \mid r \in E[3]\right\},
$$

so if $p \in E[2] \backslash\left\{o_{E}, p_{1}\right\}$, then $\mathcal{A}\left(E, \sigma_{p} \tau_{3}^{2}\right) \not \mathcal{A}\left(E, \sigma_{p_{1}} \tau_{3}^{2}\right)$. Let $p, q \in E[2] \backslash$ $\left\{o_{E}, p_{1}\right\}$ and assume that $p \neq q$. Since $E_{\tau_{3}}=\left\langle p_{1}\right\rangle$ by Proposition 3.6, $\tau_{3}(p)=q$, so it follows from Theorem 3.1 that $\mathcal{A}\left(E, \sigma_{p} \tau_{3}^{2}\right) \cong \mathcal{A}\left(E, \sigma_{q} \tau_{3}^{2}\right)$. Hence there are two Type $\mathrm{B}$ algebras up to isomorphism.

Proposition 3.11. There are two Type E algebras up to isomorphism.

Proof. Assume that $\lambda=0$. Let $q=\left(\eta^{8}: \eta^{4}: 1\right)$ where $\eta$ is a primitive 9 th root of unity.

(1) Let $\mathcal{A}\left(E, \sigma_{p} \tau_{2}^{2}\right)$ be a Type E algebra where $p \in U_{\tau_{2}^{2}} \backslash E[3]$. By Theorem 3.8 , there are $l \in \mathbb{Z}_{6}$ and $r \in E_{\tau_{2}^{2}}$ such that $p=\tau_{2}^{l}(q)+r$. By Lemma 3.2 and Proposition 3.6, $U^{\tau_{2}^{2}}=\left\langle\left(1:-\eta^{3}: 0\right)\right\rangle=E_{\tau_{2}^{2}}$, so it follows from Theorem 3.1 that $\mathcal{A}\left(E, \sigma_{p} \tau_{2}^{2}\right) \cong \mathcal{A}\left(E, \sigma_{q} \tau_{2}^{2}\right)$.

(2) Let $\mathcal{A}\left(E, \sigma_{p} \tau_{2}^{4}\right)$ be a Type E algebra where $p \in U_{\tau_{2}^{4}} \backslash E[3]$. By Theorem 3.8 , there are $l \in \mathbb{Z}_{6}$ and $r \in E_{\tau_{2}^{4}}$ such that $p=\tau_{2}^{l}(q)+r$. By Lemma 3.2 and Lemma $3.4, U^{\tau_{2}^{4}}=\left\langle\left(1:-\eta^{3}: 0\right)\right\rangle=E_{\tau_{2}^{4}}$, so it follows from Theorem 3.1 that $\mathcal{A}\left(E, \sigma_{p} \tau_{2}^{4}\right) \cong \mathcal{A}\left(E, \sigma_{q} \tau_{2}^{4}\right)$.

By Theorem $3.1, \mathcal{A}\left(E, \sigma_{q} \tau_{2}^{2}\right) \not \mathcal{A}\left(E, \sigma_{q} \tau_{2}^{4}\right)$, so there are two Type E algebras up to isomorphism.

Proposition 3.12. There are two Type $H$ algebras up to isomorphism.

Proof. Assume that $\lambda=1+\sqrt{3}$. Let $q=(1: 1: \lambda)$.

(1) Let $\mathcal{A}\left(E, \sigma_{p} \tau_{3}\right)$ be a Type $\mathrm{H}$ algebra where $p \in U_{\tau_{3}} \backslash E[3]$. By Theorem 3.8 , there exists $r \in E[3]$ such that $p=q+r$. By Lemma 3.2, $U^{\tau_{3}}=E[3]$, so it follows from Theorem 3.1 that $\mathcal{A}\left(E, \sigma_{p} \tau_{3}\right) \cong \mathcal{A}\left(E, \sigma_{q} \tau_{3}\right)$.

(2) Let $\mathcal{A}\left(E, \sigma_{p} \tau_{3}^{3}\right)$ be a Type $\mathrm{H}$ algebra where $p \in U_{\tau_{3}^{3}} \backslash E[3]$. By Theorem 3.8 , there exists $r \in E[3]$ such that $p=q+r$. By Lemma 3.2, $U^{\tau_{3}^{3}}=E[3]$, so it follows from Theorem 3.1 that $\mathcal{A}\left(E, \sigma_{p} \tau_{3}^{3}\right) \cong \mathcal{A}\left(E, \sigma_{q} \tau_{3}^{3}\right)$.

By Theorem $3.1, \mathcal{A}\left(E, \sigma_{q} \tau_{3}\right) \not \mathcal{A}\left(E, \sigma_{q} \tau_{3}^{3}\right)$, so there are two Type $\mathrm{H}$ algebras up to isomorphism. 
M. MATSUNO

We call a geometric pair $(E, \sigma)$ regular if $\mathcal{A}(E, \sigma)$ is a 3 -dimensional quadratic AS-regular algebra. The following table gives a complete list of regular geometric pairs for Type EC algebras up to isomorphism.

Theorem 3.13. (1) Every Type EC algebra is isomorphic to $\mathcal{A}(E, \sigma)$ where $(E, \sigma)$ is in Table 1.

(2) Every $(E, \sigma)$ in Table 1 is regular.

Table 1 (A list of regular geometric pairs)

\begin{tabular}{|c||c|}
\hline Type & regular geometric pairs $(E, \sigma)$ \\
\hline$A$ & $\begin{array}{c}\sigma=\sigma_{p} \\
\text { where } p=(a: b: c) \text { satisfies } a b c \neq 0 \text { and }\left(a^{3}+b^{3}+c^{3}\right)^{3} \neq(3 a b c)^{3}\end{array}$ \\
\hline \multirow{2}{*}{$B$} & $\begin{array}{c}\sigma=\sigma_{p} \tau_{1} \\
\text { where } p=(1: 1: c) \text { satisfies } c^{3}-3 \lambda c+2=0\end{array}$ \\
\hline$E$ & $\begin{array}{c}\lambda=0, \sigma=\sigma_{p} \tau_{2}^{2}, \sigma_{p} \tau_{2}^{4} \\
\text { where } p=\left(\eta^{8}: \eta^{4}: 1\right) \text { and } \eta \text { is a primitive } 9 \text { th root of unity }\end{array}$ \\
\hline$H$ & $\begin{array}{c}\lambda=1+\sqrt{3}, \quad \sigma=\sigma_{p} \tau_{3}, \sigma_{p} \tau_{3}^{3} \\
\text { where } p=(1: 1: \lambda)\end{array}$ \\
\hline
\end{tabular}

Proof. (1) By [9, Lemma 4.7], every Type EC algebra is isomorphic to $\mathcal{A}\left(E, \sigma_{p} \tau^{i}\right)$ where $p=(a: b: c) \in U_{\tau^{i}} \backslash E[3]$ and $i \in \mathbb{Z}_{|\tau|}$. If $i=0$, then $p \in E \backslash E[3]$ by Theorem 3.8, so

$$
a b c \neq 0 \text { and }\left(a^{3}+b^{3}+c^{3}\right)^{3} \neq(3 a b c)^{3} .
$$

If $i \neq 0$, then, by the proofs of Propositions 3.10, 3.11 and 3.12, $\mathcal{A}\left(E, \sigma_{p} \tau^{i}\right)$ is isomorphic to $\mathcal{A}(E, \sigma)$ where $(E, \sigma)$ is in Table 1 .

(2) By Lemma 3.3, every $(E, \sigma)$ in Table 1 is regular.

\section{Twisted superpotentials For Type EC Algebras}

In this section, we will give a list of regular twisted superpotentials for Type EC algebras. As a byproduct, we will prove that a 3-dimensional quadratic AS-regular algebra of Type EC is Calabi-Yau if and only if it is a 3-dimensional Sklyanin algebra.

For $p=(a: b: c) \in E \backslash E[3]$, we set

$$
w_{p}:=a(x y z+y z x+z x y)+b(x z y+y x z+z y x)+c\left(x^{3}+y^{3}+z^{3}\right) .
$$

Since the derivation-quotient algebra $\mathcal{D}\left(w_{p}\right)$ is a 3 -dimensional Sklyanin algebra $\mathcal{A}\left(E, \sigma_{p}\right)$, we call it a Sklyanin potential which is a regular superpotential. The next lemma is important to determine reguar twisted superpotentials.

Lemma 4.1. Let $w_{p}$ be a Sklyanin potential where $p \in E \backslash E[3]$. Assume that $\tau^{i} \in \operatorname{Aut}\left(w_{p}\right)$ where $i \in \mathbb{Z}_{|\tau|}$. Then $\left(w_{p}\right)^{\tau^{i}}$ is a regular twisted superpotential and $\mathcal{A}\left(E, \sigma_{p} \tau^{i}\right) \cong \mathcal{D}\left(w_{p}\right)^{\tau^{i}} \cong \mathcal{D}\left(\left(w_{p}\right)^{\tau^{i}}\right)$. 
Proof. Since a Sklyanin potential $w_{p}$ is a regular superpotential and $\tau^{i} \in$ Aut $\left(w_{p}\right)$ where $i \in \mathbb{Z}_{|\tau|},\left(w_{p}\right)^{\tau^{i}}$ is a regular twisted superpotential by Lemma 2.12. By [9, Theorem 4.9] and Lemma 2.10, we have that

$$
\mathcal{A}\left(E, \sigma_{p} \tau^{i}\right) \cong \mathcal{D}\left(w_{p}\right)^{\tau^{i}} \cong \mathcal{D}\left(\left(w_{p}\right)^{\tau^{i}}\right)
$$

The following table gives a complete list of regular twisted superpotentials for Type EC algebras up to isomorphism.

Theorem 4.2. (1) Every Type EC algebra is isomorphic to $\mathcal{D}(w)$ where $w$ is in Table 2.

(2) Every $w$ in Table 2 is a regular twisted superpotential.

Table 2 (A list of regular twisted superpotentials)

\begin{tabular}{|c|c|}
\hline Type & regular twisted superpotetials $w$ \\
\hline$A$ & $\begin{array}{c}a(x y z+y z x+z x y)+b(x z y+y x z+z y x)+c\left(x^{3}+y^{3}+z^{3}\right) \\
\text { where } a b c \neq 0 \text { and }\left(a^{3}+b^{3}+c^{3}\right)^{3} \neq(3 a b c)^{3}\end{array}$ \\
\hline$B$ & $\begin{array}{c}\left(x^{z}+y z x+z y^{2}\right)+\left(x z y+y^{2} z+z x^{2}\right)+c\left(x y x+y x y+z^{3}\right) \\
\text { where } c^{3}-\lambda c+2=0\end{array}$ \\
\hline & $\begin{array}{c}x z x+\eta z x^{2}+\eta^{8} x^{2} z+y x y+\eta^{4} x y^{2}+\eta^{5} y^{2} x \\
+z y z+\eta^{7} y z^{2}+\eta^{2} z^{2} y \\
\text { where } \eta \text { is a primitive } 9 \text { th root of unity. }\end{array}$ \\
\hline$E$ & $\begin{array}{c}x z x+\eta^{8} z x^{2}+\eta x^{2} z+y x y+\eta^{5} x y^{2}+\eta^{4} y^{2} x \\
+z y z+\eta^{2} y z^{2}+\eta^{7} z^{2} y \\
\text { where } \eta \text { is a primitive } 9 \text { th root of unity. }\end{array}$ \\
\hline \multirow{2}{*}{$H$} & $\begin{array}{c}\left(\varepsilon x y z+\lambda y z x+\varepsilon^{2} z x y\right)+\left(\lambda x z y+\varepsilon y x z+\varepsilon^{2} z y x\right) \\
+\left(\varepsilon \lambda x^{2} y+x y x+\varepsilon^{2} \lambda y x^{2}\right)+\left(\varepsilon^{2} x^{2} z+x z x+\varepsilon z x^{2}\right) \\
+\left(\varepsilon \lambda y^{2} x+y x y+\varepsilon^{2} \lambda y x^{2}\right)+\left(\varepsilon^{2} y^{2} z+y z y+\varepsilon z y^{2}\right) \\
+\left(z^{2} x+\lambda z x z+x z^{2}\right)+\left(z^{2} y+\lambda z y z+y z^{2}\right) \\
\quad+\left(x^{3}+y^{3}+\lambda z^{3}\right) \\
\text { where } \varepsilon \text { is a primitive } 3 \text { rd root of unity and } \lambda=1+\sqrt{3}\end{array}$ \\
\hline & 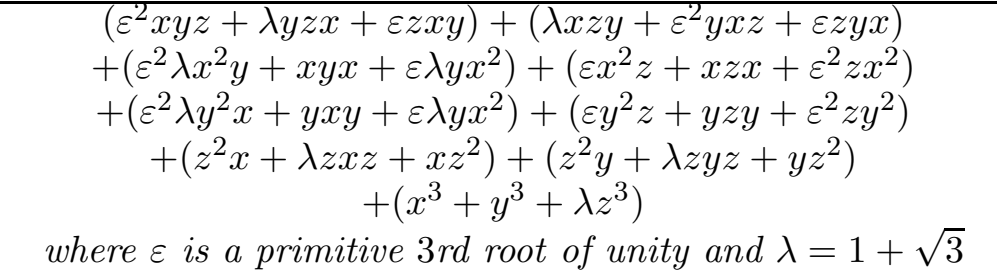 \\
\hline
\end{tabular}

Proof. To give a complete list of regular twisted superpotentials, it is enough to find a twisted superpotential $w$ such that $\mathcal{A}(E, \sigma) \cong \mathcal{D}(w)$ for each $(E, \sigma)$ in Table 1. If $\mathcal{A}\left(E, \sigma_{p} \tau^{i}\right)$ is not of Type E, then we will show that $\tau^{i} \in$ Aut $\left(w_{p}\right)$ so that $\left(w_{p}\right)^{\tau^{i}}$ is a regular twisted superpotential by Lemma 4.1 and potentials in Table 2 are given by $\left(w_{p}\right)^{\tau^{i}}$.

(i) Type A. Since a Sklyanin potential $w_{p}$ is a superpotential, the result follows. 
(ii) Type B. Let $p=(1: 1: c) \in E[2] \backslash\left\{o_{E}\right\}$. Since $\left(\tau_{1} \otimes \tau_{1} \otimes \tau_{1}\right)\left(w_{p}\right)=$ $(y x z+x z y+z y x)+(y z x+x y z+z x y)+c\left(x^{3}+y^{3}+z^{3}\right)=w_{p}, \tau_{1} \in$ Aut $\left(w_{p}\right)$. By Lemma 4.1,

$$
\begin{aligned}
\left(w_{p}\right)^{\tau_{1}} & =\left(\mathrm{id} \otimes \tau_{1} \otimes \mathrm{id}\right)\left(w_{p}\right) \\
& =\left(x^{2} z+y z x+z y^{2}\right)+\left(x z y+y^{2} z+z x^{2}\right)+c\left(x y x+y x y+z^{3}\right)
\end{aligned}
$$

is a desired regular twisted superpotential.

(iii) Type E. Let

$w=\left(x z x+\eta z x^{2}+\eta^{8} x^{2} z\right)+\left(y x y+\eta^{4} x y^{2}+\eta^{5} y^{2} x\right)+\left(z y z+\eta^{7} y z^{2}+\eta^{2} z^{2} y\right)$.

By taking the left derivatives, we have that

$$
\left\{\begin{array}{l}
\partial_{x}(w)=z x+\eta^{8} x z+\eta^{4} y^{2} \\
\partial_{y}(w)=x y+\eta^{5} y x+\eta^{7} z^{2} \\
\partial_{z}(w)=\eta x^{2}+y z+\eta^{2} z y
\end{array}\right.
$$

By taking the right derivatives, we have that

$$
\left\{\begin{array}{l}
(w) \partial_{x}=x z+\eta z x+\eta^{5} y^{2}=\eta^{8} \partial_{x}(w) \\
(w) \partial_{y}=y x+\eta^{4} x y+\eta^{2} z^{2}=\eta^{5} \partial_{y}(w), \\
(w) \partial_{z}=\eta^{8} x^{2}+z y+\eta^{7} y z=\eta^{2} \partial_{z}(w)
\end{array}\right.
$$

By Lemma 2.8, the potential $w$ is a twisted superpotential. By $[9$, Theorem 4.9], we have that $\mathcal{D}(w)=\mathcal{A}\left(E, \sigma_{q} \tau_{2}^{2}\right)$ where $q=\left(\eta^{8}: \eta^{4}: 1\right)$.

Let

$w=\left(x z x+\eta^{8} z x^{2}+\eta x^{2} z\right)+\left(y x y+\eta^{5} x y^{2}+\eta^{4} y^{2} x\right)+\left(z y z+\eta^{2} y z^{2}+\eta^{7} z^{2} y\right)$.

By taking the left derivatives, we have that

$$
\left\{\begin{array}{l}
\partial_{x}(w)=z x+\eta x z+\eta^{5} y^{2} \\
\partial_{y}(w)=x y+\eta^{4} y x+\eta^{2} z^{2} \\
\partial_{z}(w)=\eta^{8} x^{2}+y z+\eta^{7} z y .
\end{array}\right.
$$

By taking the right derivatives, we have that

$$
\left\{\begin{array}{l}
(w) \partial_{x}=x z+\eta^{8} z x+\eta^{4} y^{2}=\eta^{8} \partial_{x}(w), \\
(w) \partial_{y}=y x+\eta^{5} x y+\eta^{7} z^{2}=\eta^{5} \partial_{y}(w,) \\
(w) \partial_{z}=\eta x^{2}+z y+\eta^{2} y z=\eta^{2} \partial_{z}(w) .
\end{array}\right.
$$

By Lemma 2.8, the potential $w$ is a twisted superpotential. By $[9$, Theorem 4.9], we have that $\mathcal{D}(w)=\mathcal{A}\left(E, \sigma_{q^{\prime}} \tau_{2}^{4}\right)$ where $q^{\prime}=\left(\eta: \eta^{5}: 1\right)$. Since $q^{\prime}=\tau_{2}(q)$, it follows from Theorem 3.1 that $\mathcal{D}(w) \cong \mathcal{A}\left(E, \sigma_{q} \tau_{2}^{4}\right)$. (iv) Type H. Let $p=(1: 1: \lambda)$ where $\lambda=1+\sqrt{3}$. Since

$$
\begin{aligned}
& \left(\tau_{3} \otimes \tau_{3} \otimes \tau_{3}\right)\left(w_{p}\right) \\
& =3 \sqrt{3}(x y z+y z x+z x y)+3 \sqrt{3}(x z y+y x z+z y x)+3(3+\sqrt{3})\left(x^{3}+y^{3}+z^{3}\right) \\
& =3 \sqrt{3} w_{p}
\end{aligned}
$$


we have that $\tau_{3} \in \operatorname{Aut}\left(w_{p}\right)$. By Lemma 4.1,

$$
\begin{aligned}
\left(w_{p}\right)^{\tau_{3}} & =\left(\tau_{3}^{2} \otimes \tau_{3} \otimes \mathrm{id}\right)\left(w_{p}\right) \\
& =\left(\varepsilon x y z+\lambda y z x+\varepsilon^{2} z x y\right)+\left(\lambda x z y+\varepsilon y x z+\varepsilon^{2} z y x\right)+\left(\varepsilon \lambda x^{2} y+x y x+\varepsilon^{2} \lambda y x^{2}\right) \\
& +\left(\varepsilon^{2} x^{2} z+x z x+\varepsilon z x^{2}\right)+\left(\varepsilon \lambda y^{2} x+y x y+\varepsilon^{2} \lambda y x^{2}\right)+\left(\varepsilon^{2} y^{2} z+y z y+\varepsilon z y^{2}\right) \\
& +\left(z^{2} x+\lambda z x z+x z^{2}\right)+\left(z^{2} y+\lambda z y z+y z^{2}\right)+\left(x^{3}+y^{3}+\lambda z^{3}\right)
\end{aligned}
$$

is a desired regular twisted superpotential.

Since $\tau_{3} \in \operatorname{Aut}\left(w_{p}\right), \tau_{3}^{3} \in \operatorname{Aut}\left(w_{p}\right)$. By Lemma 4.1,

$$
\begin{aligned}
\left(w_{p}\right)^{\tau_{3}^{3}} & =\left(\tau_{3}^{2} \otimes \tau_{3}^{3} \otimes \mathrm{id}\right)\left(w_{p}\right) \\
& =\left(\varepsilon^{2} x y z+\lambda y z x+\varepsilon z x y\right)+\left(\lambda x z y+\varepsilon^{2} y x z+\varepsilon z y x\right)+\left(\varepsilon^{2} \lambda x^{2} y+x y x+\varepsilon \lambda y x^{2}\right) \\
& +\left(\varepsilon x^{2} z+x z x+\varepsilon^{2} z x^{2}\right)+\left(\varepsilon^{2} \lambda y^{2} x+y x y+\varepsilon \lambda y x^{2}\right)+\left(\varepsilon y^{2} z+y z y+\varepsilon^{2} z y^{2}\right) \\
& +\left(z^{2} x+\lambda z x z+x z^{2}\right)+\left(z^{2} y+\lambda z y z+y z^{2}\right)+\left(x^{3}+y^{3}+\lambda z^{3}\right)
\end{aligned}
$$

is a desired regular twisted superpotential.

It is easy to check that a potential $w$ in Table 2 is a superpotential if and only if it is of Type A, so the following corollary follows from Lemma 2.11 and Theorem 4.2.

Corollary 4.3. A 3-dimensional quadratic AS-regular algebra of Type EC $\mathcal{A}\left(E, \sigma_{p} \tau^{i}\right)$ is Calabi-Yau if and only if $i=0$, that is, it is a 3-dimensional Sklyanin algebra.

For a Sklyanin potential $w_{p}$, we define

$$
\operatorname{PAut}\left(w_{p}\right):=\operatorname{Aut}\left(w_{p}\right) /\left(k^{\times} \operatorname{id}_{V}\right)
$$

where $k^{\times} \operatorname{id}_{V}:=\left\{\alpha \operatorname{id}_{V} \mid \alpha \in k^{\times}\right\}$.

Lemma 4.4. Let $A=T(V) /(R)=\mathcal{A}(E, \sigma)$ be a geometric algebra. Suppose that there is a superpotential $w \in V^{\otimes 3}$ such that $A=\mathcal{D}(w)$. If $\theta \in \operatorname{Aut}(w)$, then $\overline{\theta^{*}} \in \operatorname{Aut}_{k}\left(\mathbb{P}\left(V^{*}\right), E\right)$.

Proof. Let $\theta \in \operatorname{Aut}(w)$. By [13, Lemma 3.1], $\theta$ induces a graded algerba automorphism $\phi$ of $A$. By [11, Lemma 6.4], $\phi$ induces an automorphism $\overline{\theta^{*}}=\overline{\left(\left.\phi\right|_{V}\right)^{*}} \in \operatorname{Aut}_{k}\left(\mathbb{P}\left(V^{*}\right), E\right)$.

It is easy to check the following lemma.

Lemma 4.5. Let $E$ be an elliptic curve in $\mathbb{P}^{2}, p_{1}=(1:-\varepsilon: 0)$ and $p_{2}=(1: 0:-1) \in E[3]$ where $\varepsilon$ is a primitive $3 r d$ root of unity. Then $E[3]=\left\{m p_{1}+n p_{2} \mid 0 \leq m, n \leq 2\right\}$.

We denote by $T[3]$ the set of translations by $p \in E[3]$.

Lemma 4.6. For any $p \in E \backslash E[3], T[3] \leq \operatorname{PAut}\left(w_{p}\right)$. 
Proof. Let $p=(a: b: c) \in E \backslash E[3]$. Since $\sigma_{p_{1}}=\left(\begin{array}{ccc}\varepsilon^{2} & 0 & 0 \\ 0 & 1 & 0 \\ 0 & 0 & \varepsilon\end{array}\right)$ and $\sigma_{p_{2}}=$ $\left(\begin{array}{lll}0 & 1 & 0 \\ 0 & 0 & 1 \\ 1 & 0 & 0\end{array}\right)$ where $p_{1}=(1:-\varepsilon: 0)$ and $p_{2}=(1: 0:-1)$

$$
\left(\sigma_{p_{1}} \otimes \sigma_{p_{1}} \otimes \sigma_{p_{1}}\right)\left(w_{p}\right)=w_{p}
$$

and

$$
\begin{aligned}
\left(\sigma_{p_{2}} \otimes \sigma_{p_{2}} \otimes \sigma_{p_{2}}\right)\left(w_{p}\right) & =a(y z x+z x y+x y z)+b(y x z+z y x+y x z)+c\left(y^{3}+z^{3}+x^{3}\right) \\
& =w_{p},
\end{aligned}
$$

so $\sigma_{p_{1}}, \sigma_{p_{2}} \in \operatorname{PAut}\left(w_{p}\right)$. By Lemma $4.5, T[3] \leq \operatorname{PAut}\left(w_{p}\right)$.

Proposition 4.7. Let $p \in E \backslash E[3]$.

(1) If $j(E) \neq 0,12^{3}$, then

$$
\operatorname{PAut}\left(w_{p}\right)= \begin{cases}T[3] & \text { if } p \notin E[2], \\ T[3] \rtimes\left\langle\tau_{1}\right\rangle & \text { if } p \in E[2] .\end{cases}
$$

(2) If $\lambda=0$, then

$$
\operatorname{PAut}\left(w_{p}\right)= \begin{cases}T[3] & \text { if } p \notin E[2], \\ T[3] \rtimes\left\langle\tau_{2}^{3}\right\rangle & \text { if } p \in E[2] .\end{cases}
$$

(3) If $\lambda=1+\sqrt{3}$, then

$$
\text { PAut }\left(w_{p}\right)= \begin{cases}T[3] & \text { if } p \notin E[2], \\ T[3] \rtimes\left\langle\tau_{3}^{2}\right\rangle & \text { if } p \in E[2] \backslash\left\{o_{E},(1: 1: \lambda)\right\}, \\ T[3] \rtimes\left\langle\tau_{3}\right\rangle & \text { if } p=(1: 1: \lambda) .\end{cases}
$$

Proof. Let $p=(a: b: c) \in E \backslash E[3]$.

(1) Since

$\left(\tau_{1} \otimes \tau_{1} \otimes \tau_{1}\right)\left(w_{p}\right)=a(y x z+x z y+z y x)+b(y z x+x y z+z x y)+c\left(y^{3}+x^{3}+z^{3}\right)$,

$\tau_{1} \in \operatorname{PAut}\left(w_{p}\right)$ if and only if $a=b$ if and only if $p \in E[2]$ by Lemma 2.16 .

(2) Assume that $\lambda=0$. Since $\tau_{2}^{3}=\tau_{1}, \tau_{2}^{3} \in \operatorname{PAut}\left(w_{p}\right)$ if and only if $p \in E[2]$. Since

$$
\begin{aligned}
& \left(\tau_{2} \otimes \tau_{2} \otimes \tau_{2}\right)\left(w_{p}\right) \\
& =a \eta^{3}(y x z+x z y+z y x)+b \eta^{3}(y z x+x y z+z x y)+c\left(y^{3}+x^{3}+z^{3}\right), \\
& \left(\tau_{2}^{2} \otimes \tau_{2}^{2} \otimes \tau_{2}^{2}\right)\left(w_{p}\right) \\
& =a \eta^{6}(x y z+y z x+z x y)+b \eta^{6}(x z y+y x z+z y x)+c\left(x^{3}+y^{3}+z^{3}\right),
\end{aligned}
$$

we have that $\tau_{2}, \tau_{2}^{2} \notin \operatorname{PAut}\left(w_{p}\right)$. 
(3) Assume that $\lambda=1+\sqrt{3}$. Since $\tau_{3}^{2}=\tau_{1}, \tau_{3}^{2} \in \operatorname{PAut}\left(w_{p}\right)$ if and only if $p \in E[2]$. Since

$$
\begin{aligned}
& \left(\tau_{3} \otimes \tau_{3} \otimes \tau_{3}\right)\left(w_{p}\right) \\
& =3\left(a \varepsilon+b \varepsilon^{2}+c\right)(x y z+y z x+z x y)+3\left(a \varepsilon^{2}+b \varepsilon+c\right)(x z y+y x z+z y x) \\
& +3(a+b+c)\left(x^{3}+y^{3}+z^{3}\right) \\
& \quad \tau_{3} \in \operatorname{PAut}\left(w_{p}\right) \text { if and only if } \\
& \quad \tau_{3}^{3}(p)=\left(a \varepsilon+b \varepsilon^{2}+c: a \varepsilon^{2}+b \varepsilon+c: a+b+c\right)=(a: b: c)=p
\end{aligned}
$$

if and only if $p \in E_{\tau_{3}^{3}}$. By Proposition $3.8, E_{\tau_{3}^{3}}=\langle(1: 1: \lambda)\rangle$, so if $p \neq o_{E}$, then $p \in E_{\tau_{3}^{3}}$ if and only if $p=(1: 1: \lambda)$. Thus we have that $\tau_{3} \in \operatorname{Aut}\left(w_{p}\right)$ if and only if $p=(1: 1: \lambda)$.

Let $p \in E \backslash E[3]$. Recall that a Sklyanin algebra $\mathcal{A}\left(E, \sigma_{p}\right)$ is a 3-dimensional quadratic Calabi-Yau AS-regular algebra by Corollary 4.3. Finally, we prove Theorem 1.1 in Introduction.

Theorem 4.8. Let $A$ be a 3-dimensional quadratic AS-regular algebra. Then there are a 3-dimensional quadratic Calabi-Yau AS-regular algebra $S$ and $\phi \in \operatorname{GrAut}_{k} S$ such that $A \cong S^{\phi}$ if and only if $A$ is not of Type $E$.

Proof. If $A$ is not a Type EC algebra, then the result follows from [10, Theorem 4.4], so we assume that $A$ is a Type EC algebra.

If $A$ is not of Type E, then $A \cong \mathcal{D}\left(w_{p}\right)^{\tau^{i}}$ where $\mathcal{D}\left(w_{p}\right)$ is a 3 -dimensional quadratic Calabi-Yau AS-regular algebra and $\tau^{i} \in \operatorname{Aut}\left(w_{p}\right) \subset \operatorname{GrAut}_{k} \mathcal{D}(w)$ by Lemma 2.10, the proof of Theorem 4.2 and [13, Lemma 3.1].

Conversely, let $A$ be of Type E. By Theorem 3.13 we may assume that $\lambda=0$ and $A=\mathcal{A}\left(E, \sigma_{q} \tau_{2}^{2 i}\right)$ where $q=\left(\eta^{8}: \eta^{4}: 1\right), \eta$ is a primitive 9 th root of unity and $i=1,2$. Assume that there are a 3-dimensional quadratic Calabi-Yau AS-regular algebra $S$ and $\phi \in \mathrm{GrAut}_{k} S$ such that $A \cong S^{\phi}$. Since $S=\mathcal{D}\left(w_{p}\right)$ by Corollary 4.3, we have that $\left.\phi\right|_{V} \in \operatorname{Aut}\left(w_{p}\right)$ by Lemma 2.10. By Proposition 4.7, there are $r \in E[3]$ and $j=0,1$ such that $\overline{\left(\left.\phi\right|_{V}\right)^{*}}=\sigma_{r} \tau_{2}^{3 j}$. It follows from [9, Proposition 2.6] that $\mathcal{D}\left(w_{p}\right)^{\phi}=$ $\mathcal{A}\left(E, \sigma_{p} \overline{\left(\left.\phi\right|_{V}\right)^{*}}\right)=\mathcal{A}\left(E, \sigma_{p+r} \tau_{2}^{3 j}\right)$. By Theorem 3.1, $A \neq \mathcal{A}\left(E, \sigma_{p+r} \tau_{2}^{3 j}\right)$, a contradiction.

Remark 4.9. Since every Type EC algebra is graded Morita equivalent to a Calabi-Yau AS-regular algebra by [10, Theorem 4.4], a Type E algebra is a twist of a Calabi-Yau AS-regular algebra by a twisting system introduced by [17], but not by a graded algebra automorphism by Theorem 4.8 .

\section{ACKNOWLEDGMENTS}

I am grateful to my supervisor Izuru Mori for his advice and helpful discussions. 


\section{REFERENCES}

[1] M. Artin and W. Schelter, Graded algebras of global dimension 3, Adv. Math. 66 (1987), 171-216.

[2] M. Artin, J. Tate and M. Van den Bergh, Some algebras associated to automorphisms of elliptic curves, The Grothendieck Festschrift, vol. 1, Progress in Mathematics vol. 86 (Brikhäuser, Basel, 1990) 33-85.

[3] R. Bocklandt, Graded Calabi Yau algebras of dimension 3, J. Pure Appl. Algebra 212 (2008), no. 1, 14-32.

[4] R. Bocklandt, T. Schedler and M. Wemyss, Superpotentials and higher order derivations, J. Pure Appl. Algebra 214 (2010), 1501-1522.

[5] M. Dubois-Violette, Multilinear forms and graded algebras, J. Algebra 317 (2007), 198-225. MR2360146 (2010e:16011)

[6] H. R. Frium, The group law on elliptic curves on Hesse form, Finite fields with applications to coding theory, cryptography and related areas (Oaxaca, 2001), 123151, Springer, Berlin, 2002.

[7] V. Ginzburg, Calabi-Yau algebras, arXiv:0612139 (2007).

[8] R. Hartshorne, Algebraic Geometry, Graduate Texts in Mathematics, No. 52, Springer-Verlag, New York-Heidelberg, 1977.

[9] A. Itaba and M. Matsuno, Defining relations of 3-dimensional quadratic AS-regular algebras, to appear in Mathematical Journal of Okayama Univ., (arXiv:1806.04940).

[10] A. Itaba and M. Matsuno, AS-regularity of geometric algebras of plane cubic curves, arXiv:1905.02502.

[11] I. Mori, Co-point modules over Koszul algebras, J. London Math. Soc 74 (2006), 639-656.

[12] I. Mori, Non commutative projective schemes and point schemes, Algebras, Rings and Their Representations, World Sci. Hackensack, N. J., (2006), 215-239.

[13] I. Mori and S. P. Smith, m-Koszul Artin-Schelter regular algebras, J. Algebra. 446 (2016), 373-399.

[14] I. Mori and S. P. Smith, The classification of 3-Calabi-Yau algebras with 3 generators and 3 quadratic relations, Math. Z. 287 (2017), no. 1-2, 215-241.

[15] I. Mori and K. Ueyama, Graded Morita equivalences for geometric AS-regular algebras, Glasg. Math. J. 55 (2013), no. 2, 241-257.

[16] M. Reyes, D. Rogalski and J. J. Zhang, Skew Calabi-Yau algebras and homological identities, Adv. Math. 264 (2014), 308-354.

[17] J. J. Zhang, Twisted graded algebras and equivalences of graded categories, Proc. Lond. Math. Soc., 72, (1996), 281-311.

Graduate School of Integrated Science and Technology, Shizuoka UniverSiTy, OHYA 836, SHIZUOKA 422-8529, JAPAN

E-mail address: matsuno.masaki.14@shizuoka.ac.jp 